\title{
Finite Element Modelling of a Novel Anterior Cruciate Ligament Repairing Device
}

\author{
A.Vairis ${ }^{1, *}$, M.Petousis ${ }^{1}$, N.Vidakis $^{1}$, G.Stefanoudakis ${ }^{2}$ and B.Kandyla ${ }^{3}$ \\ ${ }^{1}$ Department of Mechanical Engineering, TEI of Crete, Heraklion, 71004 Greece \\ ${ }^{2}$ Orthopaedic Surgeon, Heraklion, Greece \\ ${ }^{3}$ Internist MD, Athens, Greece
}

Received 21 May 2012; Accepted 19 March 2013

\begin{abstract}
The knee anterior cruciate ligament which connects the femur to the tibia is often torn during sudden twisting motions resulting in knee instability with surgery being an effective treatment where the torn ligament is replaced with a graft. This study provides qualitative stress information on a restored knee which has been repaired using a novel device. This device has been designed to reduce graft damage and to minimize post-surgery complications. The device as well as the intact knee have been modelled in $3 \mathrm{D}$ and studied using finite elements to assess the mechanical behaviour of the device under different loads. Results are evaluated and compared to equivalent published works. They showed that high stresses appear where tendons wrap around objects like the securing pin of the knee ligament repair device, while the highest stresses are away from the repair device components indicating that the device design does not affect the graft. Developed stresses were within the tendon elastic range, and load case direction does not affect significantly the developed stresses on the circumference of tendons in the most stressed region.
\end{abstract}

Keywords: finite element modelling, knee ligament repair, tendon graft, biomechanics, anterior cruciate ligament (ACL)

\section{Introduction}

The human knee joint has a three dimensional geometry with multiple body articulations that produce complex mechanical responses under loads that occur in everyday life and sports activities. The necessary knee joint compliance and stability for optimal daily function are provided by various menisci, ligaments as well as muscle forces. Therefore, knowledge of the complex mechanical interactions of these load bearing structures is helpful in evaluating the treatment of relevant diseases and designing assisting devices.

The ligaments provide passive support of the knee joint, while the dynamic stability of the joint is provided by muscular movements, with injury or damage to any of these load bearing structures will lead to degradation or loss of the joint function. In this joint the anterior cruciate ligament (ACL) plays an important role in maintaining normal knee function [1], and injuries to the ACL are commonly treated with surgical reconstruction as they result in joint instability in the anterioposterial direction. Active lifestyle choices, which the expanding choice of sports activities has brought, have increased dramatically the incidence of ACL ruptures. It has been reported that there are almost 100,000 cases in USA alone [2], making the ACL and its treatment techniques and assisting devices as one of the most reported orthopaedic subjects. The goal of ligament reconstruction is to replace as much of the function of the absent ligament as possible and to replicate the stability imparted by the native structure, while minimizing the change to the overall

\footnotetext{
*E-mail address: vairis@staff.teicrete.gr
}

ISSN: 1791-2377 @ 2013 Kavala Institute of Technology. All rights reserved. kinematics of the knee joint. At the same time the assisting mechanical devices (screws, pins etc.) used for this surgery need to be made of biocompatible materials and anchor the tendon grafts to the bones securely for a very long time.

The mechanical behaviour of this important structure has been studied experimentally [3-5] but as the ligament mechanical properties are different for live subjects to cadavers these studies provide reference values for the stresses and strains. Various applications in biomechanics have long demonstrated that realistic mathematical modelling is an appropriate tool for the simulation and analysis of complex biological and physical structures such as the human knee joint although they cannot be fully validated [6] due to material properties having a wide range of values, compared to man made materials where material properties can be taken to have a set value and the complex geometry of the systems modelled. During the past two decades, a number of analytical model studies with different degrees of sophistication and accuracy, have been presented in literature [7-12]. An alternative approach to in vivo measurement of structural behaviour of the body and artificial structures that are incorporated in the knee is to calculate ligament forces using numerical modelling. Previous attempts to model the ACL mechanics of ACL employing a computer model, have assumed different approaches, either where the ligament is behaving as a bundle of multiple fibres with a non-isometric behaviour [13], or not [14].

During the surgical reconstruction of injuries of the anterior or the posterior cruciate ligaments, the surgeon uses a single bundle graft to form a two bundle graft by wrapping it around the securing pin at one end and then securing it at the other end. This graft arrangement mimics the 
anteromedial and the posterolateral bundles of the ACL in the human knee [2] providing a two bundle graft of ligament with the same material properties and it has been suggested that is preferable as an arrangement compared to single fibre operation [15]. Grafts can be either biological or artificial. If a biological graft is selected, the surgeon employs the intermediate step to take the graft from another area of the body of the patient.

Conventional reconstruction protocols employ numerous fixation methods and devices for grafts [16] that may have disadvantages. The most serious of these disadvantages is graft wounding at the graft fixation points in the osteal tunnel. This appears due to the complex stress conditions that exist at the entry and exit points, which may combine tension, torsion and shearing.

Our research team of engineers and medical doctors developed an innovative ligament fixation system $[17 ; 18]$, which can be applied to biological, synthetic or hybrid grafts. This novel knee ligament repair device aims to reduce the time necessary for the operation as well as to reduce time needed to full recovery, reduce the probability of injury to the graft and bone (eg. osteolysis) during fixation, as well as to minimise recrudescence. The main drawback of the existing anterior cruciate ligament repair devices is that the securing pin is directly attached to the graft in order to fix it to the knee, bringing in effect the securing pin threads in contact with the graft causing wounding and tearing to the graft with their sharp angled edges. In the patented design employed in this study an intermediate part has been added, between the securing pin and the graft, onto which the securing pin is screwed, in order to secure the graft, without being in direct contact to (Fig. 1). This intermediate part is in touch with the graft, when it is secured in the knee, and has curvilinear surfaces to minimize the possibility of wounding the graft.

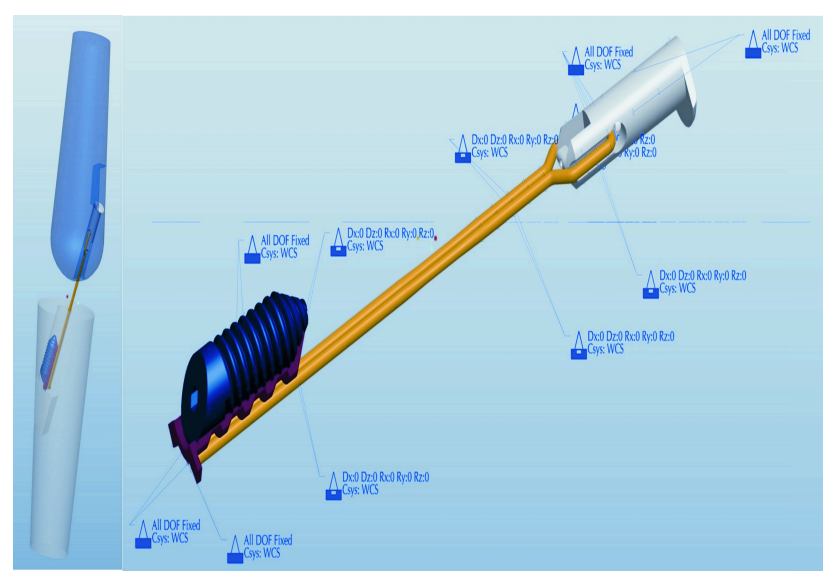

Fig. 1 Three dimensional geometrical models of the knee ligament repair device. In the right figure the constrains apllied to the knee ligament repair device for the implementation of this study are shown

The objective of this paper is to model this novel device using numerical tools. The device and the, as well as the intact knee, have been modelled in $3 \mathrm{D}$ for evaluation purposes and analysed using finite elements to assess the mechanical behaviour of the device under static load.

\section{Materials \& Methods}

During the product development phase, 3D geometrical models were developed using a CAD system (Fig. 1). These models were necessary for the visualisation of the device parts by non engineers and the accurate description of their geometry for manufacturing reasons. In order to further verify the device geometry, the $3 \mathrm{D}$ geometrical models were produced as 3D physical mock-ups. These physical mockups helped the product development team and especially the people with medical background to validate the device design and to further improve it to meet the specifications they were built to [19].

The 3D geometrical models developed (Fig. 1), were employed in the current study to determine the mechanical behaviour under static loading of this knee reconstruction device. The finite element model consists of six different 3D geometrical entities: two entities for the securing part, the security pin, the tendon graft, the tibia bone and the femur bone. The tendon graft was modelled as a string wrapped around the securing pin and secured at the other end with a screw, which it is the expected arrangement after the knee reconstruction operation. Bones were modelled as truncated cones with a diagonal hole in the direction of the drilled hole for the insertion of the graft. These models were assembled at the relative positions they have after the knee reconstruction operation is completed with the patient standing up. The image in the right part of figure 1 shows the knee reconstruction device with the bones removed so as not to hinder the view but present in the numerical analysis.

For the analysis of the model, the commercially available software package PTC Pro Mechanica was used. The geometric models were discretized using 15,457 three dimensional solid elements, and material properties were assigned to them. Three different materials (Table 1) were used, human bone for the tibia and the femur, human tendon for the graft and the biocompatible titanium alloy (Ti6A14V) for the securing device and pin.

Following this, full constrains were applied to all parts (Fig. 1), except for the graft, i.e. bones were not allowed to move or rotate in any direction. The graft constraints were applied separately in three areas, the first being the part of the graft that is inside the tibia, the second being the part of the graft that is inside the femur and the third the rest of the tendon graft which is between the first two bone (tibia and femur) parts in what is the knee. This arrangement was necessary to accurately simulate the behaviour of the graft inside the leg, within the limitations that finite element modelling can have i.e. model constraints were applied as absolute constraints and not relative to each other, so for example movement is restricted along the x-axis but cannot be restricted relative to the bone next to it. The two parts of the graft inside the tibia and the femur were allowed to move along the drilled hole in the bone, so constrains were applied to that effect. The part in the middle, where the knee is, was assumed to move freely in space, as it is not restricted by any body part.

Finally, a static force was applied as loading in the structure at the middle of the free part of the tendon. The magnitude of the force was $350 \mathrm{~N}$ (being half the weight of an average person, as the total weight of a person is received by two legs usually) $[25 ; 26]$. As the anterior cruciate ligament is restricting the forward movement of the tibia relative to the femur, this force was applied to the middle part of the graft in the knee, to simulate this behaviour. Five different load cases were studied. In each case the force being applied was at a different direction and strains and stresses were calculated. The direction of the force in each of the five scenarios is shown in figure $2 \mathrm{a}$ : 
(1) the force is applied horizontally (parallel to the ground),

(2) the force is applied to a 30 degree direction along a vertical axis to the left,

(3) the force is applied to a 30 degree direction along a vertical axis to the right,

(4) the force is applied to a 30 degree direction along a horizontal axis upwards, and

(5) the force is applied to a 30 degree direction along a horizontal axis downwards.

The application of these forces was for assessment purposes. In real life, loading would be much more complicated with dynamic loads being applied at these structures, changing in magnitude and direction continuously with time. As the real life structure is much more complicated as well as the loading, the results from this work cannot be directly compared. But they do provide qualitative information on the restored knee, employing forces of similar magnitude as the extreme loads that will be applied normally. Previous modelling works have studied numerically the effect of normal forces on the ACL [27], and analytically the effect of external pulse loads to the knee joint [28] but have not assessed a fixating device. The knee ligament repair device is at a 20 degree inclination to the vertical as the holes for the repair are usually drilled at this angle during operation.

An intact knee model was also developed (Fig. 2b) for comparison purposes with the knee ligament repair device. The developed finite element model for the intact knee consists of three different 3D geometrical entities: tibia bone, femur bone and ACL. Bones were modelled in a similar fashion as truncated cones while the ACL was assumed to be elliptical [29] with an inclination of 10 degrees, pointing to the posterior direction.

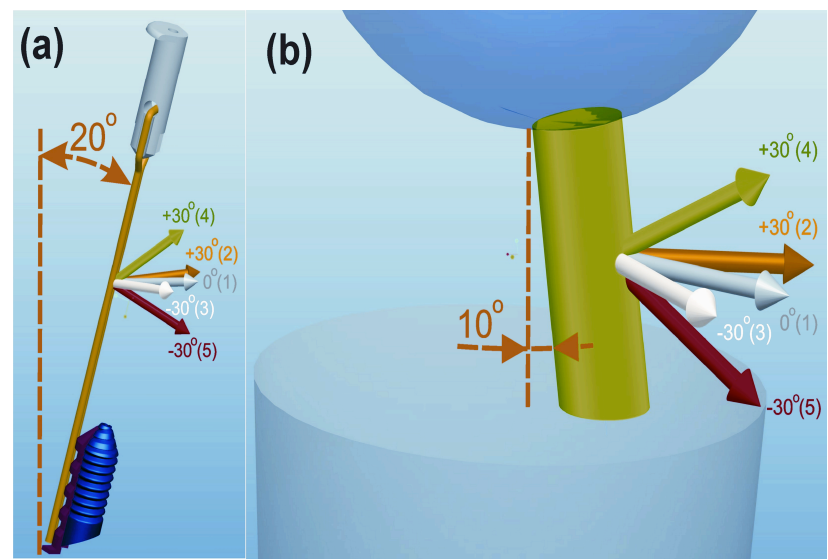

Fig. 2 Directions of the ifferent load cases studied (numbers next to the inclination angles denote the equivalent load case): (a) knee repair device, (b) intact knee model

The geometric model of the intact knee was discretized using 105 three dimensional solid elements, and material properties were assigned to these. Two different materials (Table 1) were used, ligament and bone for the tibia and the femur. Both bones were fully constrained at their ends, while the ligament was not constrained at all, as it is solely attached to the bones. The same five different load cases with the knee ligament repair device were applied to the intact knee model (Figure 2b) for comparison purposes.

Table 1. Material properties used in the analysis.

\section{Material properties}

\begin{tabular}{l|c|c}
\hline & $\begin{array}{c}\text { Young's Modulus } \\
\text { (GPa) }\end{array}$ & Poisson's Ratio \\
\hline Bone & $17[21]$ & $0.36[21]$ \\
Ti6Al4V & $113.8[20]$ & $0.34[20]$ \\
Ligament & $0.39[22]$ & $0.4[21]$ \\
Tendon graft & $1.3[23]$ & $0.46[24]$ \\
\hline
\end{tabular}

\section{Results}

Figures $3 a$ and $3 b$ show the calculated strain and stress distribution in the knee repair device model for the case where the applied force is horizontal with a 30 degree inclination to the left. The pin side of the graft developed higher strain and stress than on the other side of the graft. Figures $3 \mathrm{c}$ and $3 \mathrm{~d}$ show the calculated strain and stress distribution in the intact knee model for the same force magnitude and direction.

In all cases the distribution of strain and stress for both the knee repair device and the intact knee model were similar, while their magnitude does not change dramatically for the different load cases studied.

For the knee repair device, high strain and stress values appeared in the middle of the graft. It should be noted that the maximum values for strain and stress appear at the osteal exit points, but this should be a numerical artifact due to the geometric entity construction. In practice, this "killer corner" feature plays an important role in other orthopaedic operations like PCL reconstruction, where grafts make sharp turns [31].

For the intact knee model, higher strain and stress values appeared in the middle of the ligament, while the maximum strains and stresses appeared at the point where the ligament is connected to the bones, in every case. The femur end of the ligament developed higher strain and stress than the other end of the ligament, due to the contoured shape of the contact surface of the ligament with the femur, while the contact surface of the ligament with the tibia in the model is flat. In the former case the geometric representation of the finite element of the contoured contact between the ligament and the tibia introduces a stress concentration point. These geometric representations are necessary for the 3D model to work as expected but at the same time they introduce mechanical stresses which are related to the FE modelling intricacies, and they may not be present in the physical system. It is therefore necessary to point for the non-FE expert the limit that these models may have in explaining complex physical systems.

Table 2 shows the maximum strain and stress values calculated for all the load cases in the knee repair device model. As it is shown, the maximum stress and strain values do not significantly differ between the different load cases. Higher values were calculated for the horizontal force load case, while lower values were calculated in the load cases where the force direction was horizontal with an inclination to the left and to the right. In these two cases, the calculated strain and stress values are very close, as it is expected due to the geometry and load symmetry, which is the expected response of such a model. In the cases where the force has 
an inclination upwards and downwards, the calculated values are not expected to deviate from the ones calculated in this study, due to the initial 20 degrees inclination of the knee repair device model. The calculated strain and stress values at the securing device and the pin were significantly lower than the developed strain and stress values at the tendon graft.

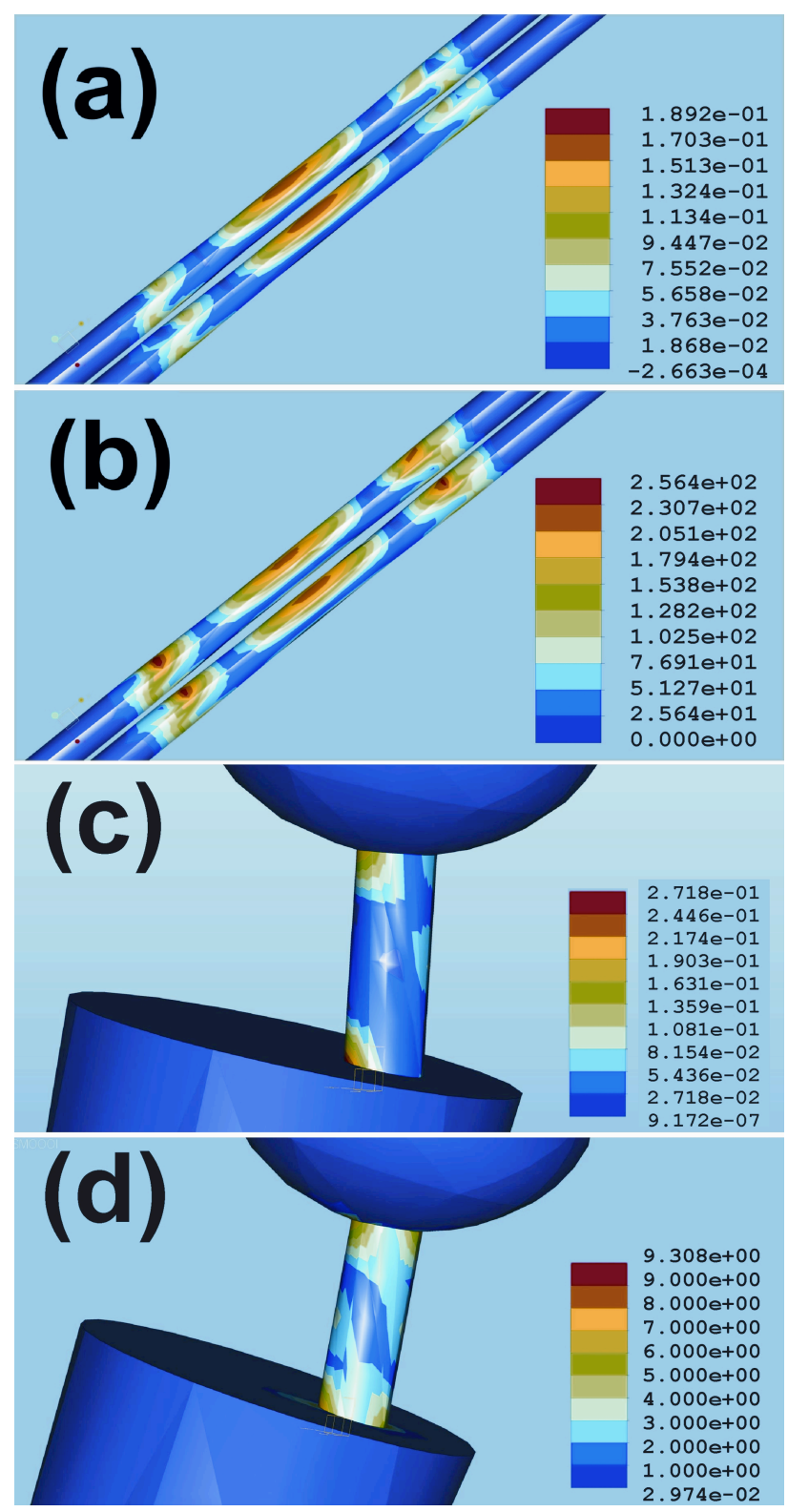

Fig. 3 Strain (a) and stress (b) distribution for the knee repair device, strain (c) and stress (d) distribution for the intact knee model (Force direction is horizontal and at 30 degrees to the left)

Table 2. Knee repair device and intact knee model maximum strain and stress for each load case.

\begin{tabular}{l|c|c|c|c|c}
\hline \multicolumn{2}{l|}{} & \multicolumn{2}{|c|}{$\begin{array}{c}\text { Knee repair device maximum } \\
\text { strain and stress for each load } \\
\text { case }\end{array}$} & \multicolumn{2}{|c}{$\begin{array}{c}\text { Intact knee model maximum } \\
\text { strain and stress for each load } \\
\text { case }\end{array}$} \\
\hline $\begin{array}{c}\text { Force } \\
\text { direction }\end{array}$ & $\begin{array}{c}\text { Load } \\
\text { case }\end{array}$ & $\begin{array}{c}\text { Maximum } \\
\text { Strain }\end{array}$ & $\begin{array}{c}\text { Maximum } \\
\text { Stress } \\
\text { (MPa) }\end{array}$ & $\begin{array}{c}\text { Maximum } \\
\text { Strain }\end{array}$ & $\begin{array}{c}\text { Maximum } \\
\text { Stress } \\
\text { (MPa) }\end{array}$ \\
\hline $\begin{array}{l}\text { Horizontal } \\
\text { 30 degrees } \\
\text { left }\end{array}$ & 1 & 0.25 & 361 & 0.274 & 8.844 \\
$\begin{array}{l}\mathbf{3 0} \text { degrees } \\
\text { right }\end{array}$ & 2 & 0.19 & 256 & 0.272 & 9.308 \\
$\begin{array}{l}\mathbf{3 0} \text { degrees } \\
\text { up }\end{array}$ & 4 & 0.19 & 257 & 0.276 & 9.592 \\
$\begin{array}{l}\text { 30degrees } \\
\text { down }\end{array}$ & 5 & 0.24 & 340 & 0.280 & 8.867 \\
\hline
\end{tabular}

Table 2 also shows the maximum strain and stress values calculated for all the load cases for the intact knee model. With this model as well, the maximum strain and stress values do not significantly differ between the different load cases. Higher values were calculated at the load case where the force is horizontal with an inclination of 30 degrees to the right. The values calculated for the load case where the force is horizontal with an inclination of 30 degrees to the left, are similar. Lower values were calculated in the load case where the force was applied to a 30 degree direction downwards along a horizontal axis. This is expected, due to the original 10 degrees inclination of the ACL to the posterior direction, which increases the load component in the longitudinal axis of the ACL in this load case. This behaviour is different compared to the knee repair device model, due to the initial 20 degrees inclination of the latter in a vertical to the intact knee model direction and the complex geometry of the knee repair device model.

In addition to the above, the strain and stress distribution on the circumference of the graft in the middle of the graft, close to the area where the maximum strain and stress appears, was studied for the knee repair device model (see figures $4 \mathrm{a}$ and $4 \mathrm{~b}$ ) for each of the five different load cases. As it is shown, the distribution of strain and stress is similar for all the different load cases, with the values being much lower than the maximum values calculated for the whole device case. Also, in the cases in which the force is horizontal with an inclination to the left and to the right, the distribution of strain and stress is almost symmetrical about the equivalent average value axis for each graph. So, the points on the circumference of the graft that have higher strain and stress values in the right force load case have lower values in the left force load case and the opposite.

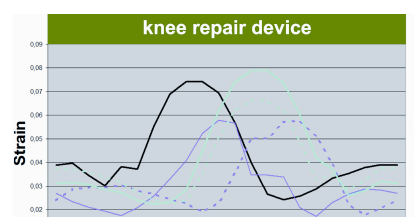

(a)

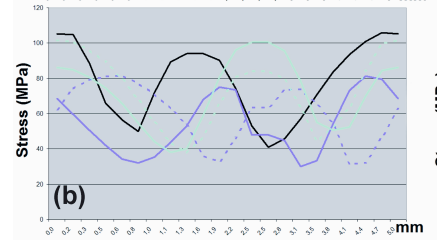

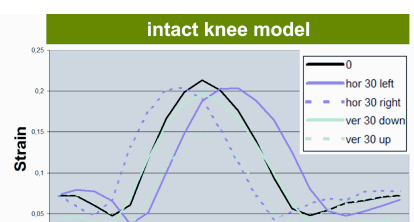

(c)

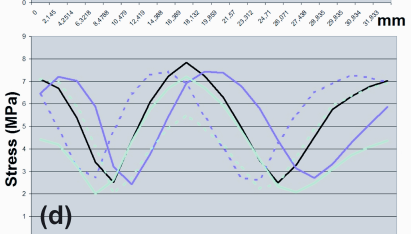

(d)
Fig. 4 (a) Strain and (b) stress distribution along the circumference of the tendon graft in the knee repair device model, close to the area where the maximum stress appears for the five load cases (middle of the graft). (c) Strain and (d) stress distribution along the circumference of the ligament in the intact knee model, close to the area where the maximum stress appears for the five load cases (at the position where the ligament contacts with the femur)

Equivalent strain (Fig. 4c) and stress (Fig. 4d) distribution graphs were determined for the ligament circumference at the point where the ligament is connected with the tibia on the intact knee model for each of the five different load cases. As it is shown, the behaviour of the model and the distribution of the strain and stress values are similar as before on the knee repair device model. The calculated strain and stress distribution values for the load cases where the force was horizontal with an inclination to the left and to the right are very close, as it is expected from 
the geometry and load symmetry, which is the expected response of such a model. In the cases where the force is horizontal as well as has an inclination upwards and downwards, the distribution of strain and stress in the ligament circumference is similar, as expected, but with different maximum calculated values due to the different load directions . Finally, each of the two models equivalent strain and stress distribution values can not be directly compared, due to geometry differences which prevent the comparison of stresses and strains at exactly the same position.

\section{Discussion}

As this study is part of a larger research programme to develop a novel ligament repair device, it was observed that tendon stresses which develop in the screw part of the device are not significant for failure to occur, which would be the desired behaviour for such a device to be used in orthopaedic operations. This study follows our previous study for the calculation of the developing strain and stress values on the knee ligament repair device [32]. The results in the current study are very close to the results of the previous study, with regards to the maximum stress values in each load case and the distribution of stress values along the circumference of the graft. The same tendon graft section was studied in both studies. The strain results reported are different from the results of the previous study, due to the comprehensive material properties used in this study, where there are the appropriate material properties for the ACL and the graft. A limitation of the current study, as with most numerical studies of such physical systems, is that in real life knee loads are more complex in terms of point of application and magnitude that change with time, so the results presented can not be correlated with experimental data. Therefore a realistic aim for this study is to determine whether the change of load direction significantly affects the developed strain and stress and its stress distribution on the graft, as well as to determine the stress concentration close to the repair device, so as to assess the possibility of the repair device wounding the graft.

Comparing the maximum calculated values between the two models, it is shown that the maximum calculated strain values were similar, while the calculated maximum stress values differ by an order of magnitude, mainly due to the much larger ACL section area of the intact knee model. The intact knee has a cross section area of $86.39 \mathrm{~mm}^{2}$, while the repair device graft of $5.09 \mathrm{~mm}^{2}$, making the area of the graft about $6 \%$ of the intact knee ACL area. Again, higher strains and stresses appeared close to the bone sections, where constrains were applied, but these values were still lower than the developed strains and stresses at the ligament.

Stresses and strains calculated and their distribution with the two developed finite elements models were the expected validating in this way the models developed. High stresses were present at high curvature points where tendons wrap around objects such as the securing pin of the developed knee ligament repair device, while the highest stresses appeared further away from the repair device, showing that the design of the device does not affect the graft. In order to further verify the reliability of the developed numerical models, a sensitivity analysis was run, with load magnitude as a parameter. In each direction the simulation was run with five different force values and the linear behaviour of the models was verified for both strain and stress calculated values.

Results produced by such models cannot be validated with experimental data taken under the same conditions as the device is still under development. Experiments in literature refer mainly to the measurement of the forces and displacement in physical knee models, or in cadavers $[3 ; 5$; 26; 33-35]. Such experimental results have been employed for the verification of geometrical models developed to simulate knee joint kinematics and loads $[1 ; 8 ; 12 ; 27 ; 36]$. In literature, there are few studies available related to the simulation of the knee joint behaviour after an ACL reconstruction operation. Veselkoa and Godler [13] employed a geometrical model to study knee kinematics in different knee positions and especially the fibre length change during knee motion of peripheral fibres of original ACL and modelled grafts. The results from this study were found to be consistent with experimental findings of another study. Suggs, Wang, and Li [37] developed a geometrical model to study the anterior tibial translation, internal tibial rotation and ACL/graft tension in different knee positions, under different knee loadings. The developed geometrical model was validated with experimental data. Tsarouhas et al. [38] examined patients who were operated for ACL reconstruction employing different reconstruction techniques. Authors studied the kinematic and kinetic analysis of knee rotational stability after single- and doublebundle anterior cruciate ligament reconstruction. These works employ different methods to study the reconstructed knee joint. They are focusing on the knee joint kinematics after the ACL reconstruction and are not studying the developed loads and their distribution on the graft.

There are no validation studies available in literature for knee ligament repair devices where calculated strains or stresses are compared to experimental values. Only qualitative verification about the models reliability and validity of results can be made in a similar manner in the same way as to the one used in this study. For the knee ligament repair device, there are no clinical results available for comparison as it has not been through clinical studies. For the intact knee model, results from this study were found to be consistent to those presented in literature $[8 ; 27 ; 30]$. In these studies structures have been exposed to similar but lower loads. The stresses calculated with our model are therefore higher to the ones presented in literature as expected.

Several different material properties for tendons and ligaments have been used previously in literature, and have been estimated experimentally. But as with all natural materials there is a large deviation in material properties between various studies for the same material. Material properties are usually determined in cadaveric studies on limbs acquired from aging populations which may significantly underestimate the strength of the ACL in a young healthy adult, who is most likely to benefit from an ACL reconstruction operation. The material properties employed in the current study are taken from literature and they were chosen as the average value of those reported in several different studies (Table 1). A further limitation of this study is that it uses a linear model for the material properties of the graft and the ligament. Previous works are also using linear material properties in the finite element models for the knee joint. The estimation and application of non-linear material properties for such systems is a challenging task and can be a topic for a separate study. 


\section{Conclusions}

This study aims to model using finite elements and use the analysis results to assess the design based on the mechanical stress concentration in an ACL repair device and the graft.

A number of load case analyses were performed for both a simplified intact knee model and ACL repaired one and the following conclusions were drawn:
- The model developed has calculated stresses that were within the tendon elastic range, which would be the preferred response to such loads

- Load case direction does not affect significantly the developed stresses on the circumference of tendons in the most stressed region

- High stresses develop at points where tendons wrap around objects such as the securing pin of the knee ligament repair

- Tendon stresses in the screw part of the device are not significant for failure to occur at that point.

\section{References}

1. KE. Moglo, A. Shirazi-Adl, Knee, 10, 265 (2003)

2. T. Zantop, W. Petersen, F.H. Fu, Operative techniques in orthopaedics, 15, 20 (2005)

3. JH. Lo, O. Muller, M. Wunschel, S. Bauer, N. Wulker, J. Biomech. 41, 1855 (2008)

4. R.S. Jones, N.S. Nawana Pearcy, D.J.A. Learmonth, D.R. Bickerstaff, J.J. Costi, R.S. Paterson, Clin. Biomech, 10, 339 (1995)

5. S.L.Y. Woo, R.J. Fox, M. Sakane, G.A. Livesay, T.W. Rudy, F.H. $\mathrm{Fu}$, Knee, 5, 267 (1998)

6. M. Viceconti, S. Olsen, K. Burton, Clin. Biomech. 20, 451 (2005)

7. A. Huson, C.W. Spoor, A.J. Verbout, Acta Morphol. Neerl. - Scand. 24, 45 (1989)

8. M.Z. Bendjaballah, A. Shirazi-AdI, D.J. Zukor, Knee, 2, 69 (1995)

9. F. Bonnel, J-P. Micaleff, Surg. Radiol. Anat. 10, 221 (1988)

10. R.R. Bini, F. Diefenthaeler, C.B. Mota, J. Electromyogr. Kinesiol., 20, $102(2010)$

11. A.E. Yousif, S.R.F. Al-Ruznamachi, 25th Southern Biomedical Engineering Conference. IFMBE Proceedings, 24, 227 (2009)

12. Y. Song, R.E. Debski, V. Musahl, M. Thomas, SL-Y Woo, J. Biomech., 37, 383 (2004)

13. M. Veselkoa, I. Godler, Comput. Biol. Med., 30, 299 (2000)

14. A.A. Amis, T.D. Zavras, Knee, 2, 5 (1995)

15. F. Giron, P. Cuomo, A. Edwards, A.M.J. Bull, A.A.P. Amis, P. Aglietti, Arthroscopy, 23, 7 (2007)

16. L.S. Beasley, D.E. Weiland, A.F. Vidal, A. Chhabra, A.S. Herzka, M.T. Feng, R.V. West, Oper. Tech. Orthop ,15, 5 (2005)

17. G. Stefanoudakis, Greek Patent GR20060100567 (2008)

18. G. Stefanoudakis G Greek Patent GR20060100566 (2008)

19. M. Petousis, A. Vairis, N. Vidakis, G. Pappas, M. Koudoumas, Procs of the 6th International Conference on New Horizons in Industry, Business and Education, Santorini, Greece (2009)

20. R. Boyer, G. Welsch, and E.W. Collings, Materials Properties Handbook: Titanium Alloys. ASM International, Materials Park(1994)

21. S.C. Cowin Bone mechanics, CRC Press (1989)

22. S.L.-Y. Woo, A.J. Almarza, R. Liang, M.B. Fisher, Functional Tissue Engineering of Ligament and Tendon Injuries. Book Ch. no 9.
Translational Approaches In Tissue Engineering And Regenerative Medicine, Artech House Publisher (2007)

23. N.D. Reeves, C.N. Maganaris, N. Maffulli, J. Rittweger, Journal of Biomechanics 42, 797 (2009)

24. O. Lopez, G. Li, H. Rubash, ASME Journal of Biomechanical Engineering 123, 341 (2001)

25. G.E. Lutz, R.A. Palmitier, K.N. An, E.Y. Chao, J. Bone. Joint. Surg. 75,732 (1993)

26. D.E. Toutoungi, T.W. Lu, A. Leardini, F. Catani, J.J. O'Connor, Clin. Biomech. 15, 176 (2000)

27. W. Mesfar, A. Shirazi-Adl, Knee 12, 424 (2005)

28. V. Mosnegutu, Rev. Roum. Sci. Tech. Ser. Mec. Appl. 53, 227 (2008)

29. D.P. Pioletti, L.R. Rakotomanana, P-F. Leyvraz, Med. Eng. Phys. 21, 95 (1999)

30. D.P. Pioletti, L.R. Rakotomanana, J.F. Benvenuti, P.F. Leyvraz, J. Biomech. 31, 753 (1998)

31. K.F. Bowman, J.K Sekiya, Oper. Tech. Sports. Med., 17, 126 (2009)

32. A. Vairis, M. Petousis, N. Vidakis, G. Stefanoudakis, B. Kandyla, ProcS. IEEE 9th International Symposium on Distributed Computing and Applications To Business, Engineering \& Science (DCABES 2010), Hong Kong (2010)

33. T. Fukubayashmi, P. Torzilli, M. Sherman and R. Warren J. Bone Joint Surg, 64, 258 (1982)

34. A. Kanamori, SL-Y. Woo, B. Ma, J. Zeminski, T.W. Rudy, G. Li and G.A.Livesay The Journal of Arthroscopic and Related Surgery 16,633 (2000)

35. T.J. Withrow, L.J. Huston, E.M. Wojtys, and J.A. Ashton-Miller, The American Journal of Sports Medicine 34, 269 (2006)

36. E. Pena, B,Calvo, M.A. Martınez, M. Doblare Journal of Biomechanics 39, 1686 (2006)

37. J. Suggs, C. Wang, G. Li, Clinical Biomechanics 18, 35 (2003)

38. A. Tsarouhas, M. Iosifidis, D. Kotzamitelos, G. Spyropoulos, T. Tsatalas, and G. Giakas, The Journal of Arthroscopic and Related Surgery 26, 885 (2010) 